\title{
A coordinated phosphorylation by Lats and CK1 regulates YAP stability through $\mathrm{SCF}^{\beta-T R C P}$
}

\author{
Bin Zhao, ${ }^{1} \mathrm{Li} \mathrm{Li},{ }^{1,2}$ Karen Tumaneng, ${ }^{1}$ Cun-Yu Wang, ${ }^{3}$ and Kun-Liang Guan ${ }^{1,4}$ \\ ${ }^{1}$ Department of Pharmacology and Moores Cancer Center, University of California at San Diego, La Jolla, California 92093-0815, \\ USA; $^{2}$ Department of Biological Chemistry, University of Michigan, Ann Arbor, Michigan 48109, USA; ${ }^{3}$ Laboratory of Molecular \\ Signaling, Division of Oral Biology and Medicine, University of California at Los Angeles School of Dentistry, Los Angeles, \\ California 90095, USA
}

The Yes-associated protein (YAP) transcription coactivator is a key regulator of organ size and a candidate human oncogene. YAP is inhibited by the Hippo pathway kinase cascade, at least in part via phosphorylation of Ser 127, which results in YAP 14-3-3 binding and cytoplasmic retention. Here we report that YAP is phosphorylated by Lats on all of the five consensus HXRXXS motifs. Phosphorylation of Ser 381 in one of them primes YAP for subsequent phosphorylation by $\mathrm{CK} 1 \delta / \varepsilon$ in a phosphodegron. The phosphorylated phosphodegron then recruits the $\mathrm{SCF}^{\beta-T R C P}$ E3 ubiquitin ligase, which catalyzes YAP ubiquitination, ultimately leading to YAP degradation. The phosphodegron-mediated degradation and the Ser 127 phosphorylation-dependent translocation coordinately suppress YAP oncogenic activity. Our study identified CK1 $\mathrm{C} / \varepsilon$ as new regulators of YAP and uncovered an intricate mechanism of YAP regulation by the Hippo pathway via both S127 phosphorylation-mediated spatial regulation (nuclear-cytoplasmic shuttling) and the phosphodegron-mediated temporal regulation (degradation).

[Keywords: YAP; Hippo pathway; CK1; SCF; Lats; phosphodegron]

Supplemental material is available at http://www.genesdev.org.

Received July 14, 2009; revised version accepted November 9, 2009.

The Yes-associated protein (YAP) transcription coactivator is a key regulator of organ size (Camargo et al. 2007; Dong et al. 2007). Mutation of Yki, the Drosophila homolog of YAP, results in dramatically reduced organ size, while Yki overexpression causes tissue overgrowth (Huang et al. 2005). Similarly, transgenic overexpression of YAP in mice liver results in enlarged liver four times the size of normal control and causes liver tumors (Camargo et al. 2007; Dong et al. 2007). Consistently, yap has been identified as a candidate oncogene in human chromosome 11q22 amplicon and the equivalent genomic amplicon in a mouse hepatocellular carcinoma (HCC) model (Overholtzer et al. 2006; Zender et al. 2006). Besides the genomic amplification, YAP protein levels and nuclear localization were also shown to be elevated in multiple types of human cancers (Zender et al. 2006; Dong et al. 2007; Zhao et al. 2007; Steinhardt et al. 2008). YAP cooperates with the myc oncogene to stimulate tumor growth in vivo (Zender et al. 2006), and active YAP mutants induce oncogenic cell transformation in vitro (Zhao et al. 2009). These observations strongly indicate the function of yap as an oncogene.

${ }^{4}$ Corresponding author.

E-MAIL kuguan@ucsd.edu; FAX (858) 534-7628.

Article is online at http://www.genesdev.org/cgi/doi/10.1101/gad.1843810.
As shown above, the mechanism controlling YAP activity is obviously a key question. Regulation of YAP/ Yki activity was first revealed by genetic studies of the Hippo tumor suppressor pathway in Drosophila (KangoSingh et al. 2002; Tapon et al. 2002; Harvey et al. 2003; Jia et al. 2003; Pantalacci et al. 2003; Udan et al. 2003; S Wu et al. 2003; Lai et al. 2005). The Hippo pathway limits organ size by inhibiting cell proliferation and promoting apoptosis through inhibition of Yki (Huang et al. 2005). Biochemical studies showed that Yki is directly phosphorylated and inhibited by the Wts protein kinase, which is phosphorylated and activated by the Hippo (Hpo) protein kinase (Dong et al. 2007; Oh and Irvine 2009). Components of the Hippo pathway are highly conserved in mammals. Recent studies from our group and others (Zhao et al. 2007; Hao et al. 2008; Oka et al. 2008; Zhang et al. 2008) have demonstrated that YAP is phosphorylated and inhibited by the Lats tumor suppressor kinase, the mammalian homolog of Wts. YAP has five HXRXXS Lats phosphorylation consensus motifs, in which phosphorylation of Ser 127 results in 14-3-3 binding and cytoplasmic retention of YAP (Zhao et al. 2007). Therefore, YAP can be inhibited by spatial separation from its nuclear target transcription factors, such as TEAD family members (Zhao et al. 2008). This mechanism of YAP regulation is 
implicated in cell contact inhibition and tissue growth control (Zhao et al. 2007; Zeng and Hong 2008). However, whether the other four HXRXXS sites in YAP are phosphorylated in vivo is unknown. Nevertheless, there is evidence that at least some of these sites are important for YAP regulation because the YAP-5SA mutant, in which serine residues in all five HXRXXS motifs are mutated to alanines, is more active than YAP-S127A in promoting cell proliferation (Zhao et al. 2009). Furthermore, YAP-5SA expression can transform NIH-3T3 cells, but YAP-S127A cannot (Zhao et al. 2009). Therefore it is important to know which HXRXXS site regulates YAP activity and what the underlying mechanism is.

We previously reported cell density-dependent activation of the Hippo pathway and increase of YAP phosphorylation (Zhao et al. 2007). We also observed a dramatic cell density-dependent decline of YAP protein levels (this study), indicating a possible role of the Hippo pathway in regulating YAP protein stability. Ubiquitin-dependent proteolysis regulates protein turnover and serves key regulatory roles in various biological processes (Hershko and Ciechanover 1998). Ubiquitination is mostly catalyzed by the sequential action of three enzymes; namely, E1 (ubiquitin-activating enzyme), E2 (ubiquitin-conjugating enzymes), and E3 (ubiquitin ligase). E3 determines substrate specificity, and its substrate binding is the primary event controlling the stability of a particular protein (Pickart 2001). The SCF is a four-subunit RING-type E3 consisting of the RING domain protein Rbx1, two scaffold proteins (Cul1 and SKP1), and one of the many F-box proteins (Deshaies 1999). The F-box proteins contain various C-terminal protein-protein interaction domains that determine substrate specificity (Deshaies 1999). $\beta$-Transducin repeat-containing proteins $(\beta$-TRCP) belong to the F-box protein family with two paralogs in mammals: $\beta$-TRCP1 and $\beta$-TRCP2. They play redundant roles in the ubiquitination of well-established substrates, including $\beta$-catenin (Hart et al. 1999; Latres et al. 1999) and IкB (Li and Verma 2002). $\beta$-TRCP recognizes a DSGXXS motif in which the serine residues are phosphorylated, and therefore this motif is called phosphodegron (Fuchs et al. 2004). Phosphorylation of phosphodegron is the major mechanism regulating $\beta$-TRCP substrate binding (Fuchs et al. 2004).

In this study, we describe a new mechanism of YAP inhibition involving coordinated phosphorylation by two kinases and subsequent $\mathrm{SCF}^{\beta-\mathrm{TRCP}}$-mediated ubiquitination and degradation. We show that YAP is phosphorylated by Lats on Ser 381 in one of the HXRXXS motifs, and this phosphorylation provides the priming signal for $\mathrm{CK} 1 \delta / \varepsilon$ to phosphorylate a phosphodegron in YAP. The phosphorylated phosphodegron recruits $\beta$-TRCP, leading to YAP ubiquitination and degradation under conditions of elevated Hippo pathway activity, such as cell contact inhibition. Hence, YAP is inhibited by two mechanisms-the S127 phosphorylation-mediated spatial regulation (nuclear-cytoplasmic shuttling) and the S381 phosphorylation-mediated temporal regulation (the phosphodegron-induced degradation) - that coordinately suppress YAP oncogenic activity.

\section{Results}

YAP is phosphorylated in vivo on multiple sites, including the five Lats consensus HXRXXS motifs

YAP phosphorylation on S127 in the Lats consensus HXRXXS motif increases with cell density, and inhibits YAP activity by promoting YAP cytoplasmic retention through interaction with 14-3-3 (Zhao et al. 2007). Serines in the other four HXRXXS motifs in YAP could all be phosphorylated by Lats in vitro (Zhao et al. 2009). However, their phosphorylation in vivo has not been shown, and the function of these Lats consensus sites is unclear. To study the function of these candidate phosphorylation sites, we performed mass spectrometry analysis of Flag-SBP (streptavidin-binding peptide)-tagged YAP protein isolated from cultured cells. As shown in Figure 1A, 11 phosphopeptides were identified representing 10 unique phosphorylation sites, including all five predicted Lats phosphorylation sites (S61, S109, S127, S164, and S381). This indicates that the Last phosphorylation consensus sites are indeed phosphorylated in vivo. Phosphorylation on several other residues was also identified, including T63, S138, S289, S351, and S384 (Fig. 1A).

To further confirm phosphorylation on the HXRXXS motifs in vivo, we examined the electrophoretic mobility of various YAP phosphorylation site mutants expressed in HEK293 cells on gels containing Phos tag-conjugated acrylamide, which specifically retards phosphorylated proteins. YAP expressed in HEK293 cells migrated as multiple bands on Phos tag gel (Fig. 1B), indicating various phosphorylation states on multiple sites. However, with Mst and Lats cotransfection, wild-type YAP mostly showed as the slowest migrating form (Fig. 1B). This result suggests that the differentially migrating forms of YAP resulted mainly from phosphorylation on the Hippo pathway target sites. Consistent with the mass spectrometry data, mutation of S61 alone or in combination with S127 mutation increased YAP mobility with or without Mst and Lats cotransfection (Fig. 1B), supporting that S61 of YAP is phosphorylated by Lats in vivo. Similar effects on YAP electrophoretic mobility were observed by mutating S109 or S164 alone or in combination with other mutations (Fig. 1B), indicating that these two sites are also phosphorylated in vivo. Under Mst/Lats cotransfection, YAP-5SA exhibited a slightly faster mobility than YAP-4SA/S381 (Fig. 1B). However, in other conditions, mutation of S381 did not increase YAP mobility (Fig. 1B). This might result from sequence context-dependent interference with Phos tag binding. Nevertheless, the phosphorylation of S381 was confirmed by two different phosphorylation-specific antibodies (Supplemental Figs. S1A, S4A).

\section{Ser 127 and Ser 381 are key phosphorylation sites in suppressing YAP oncogenic activity}

We observed that YAP-5SA is more active than YAPS127A in promoting cell proliferation (Zhao et al. 2009), indicating that the other HXRXXS motifs also contribute to YAP regulation. There is also a dramatic difference between YAP-5SA and YAP-S127A mutants in oncogenic 
A

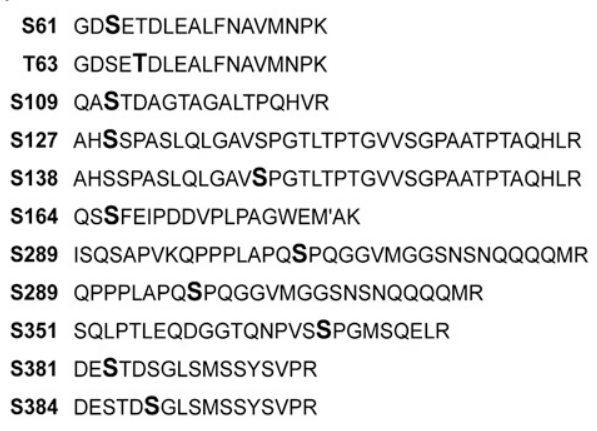

C

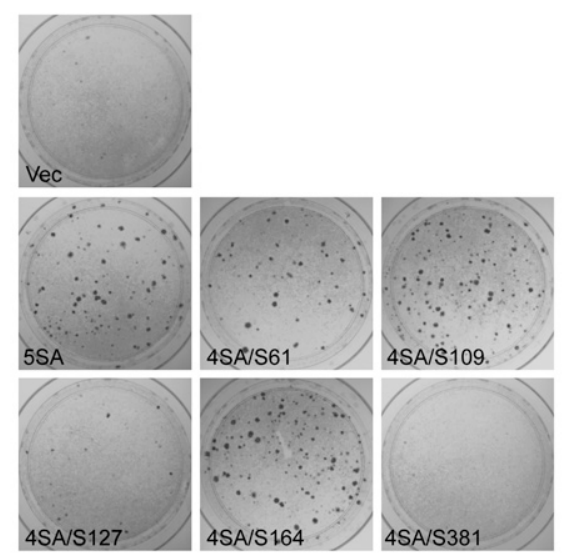

B
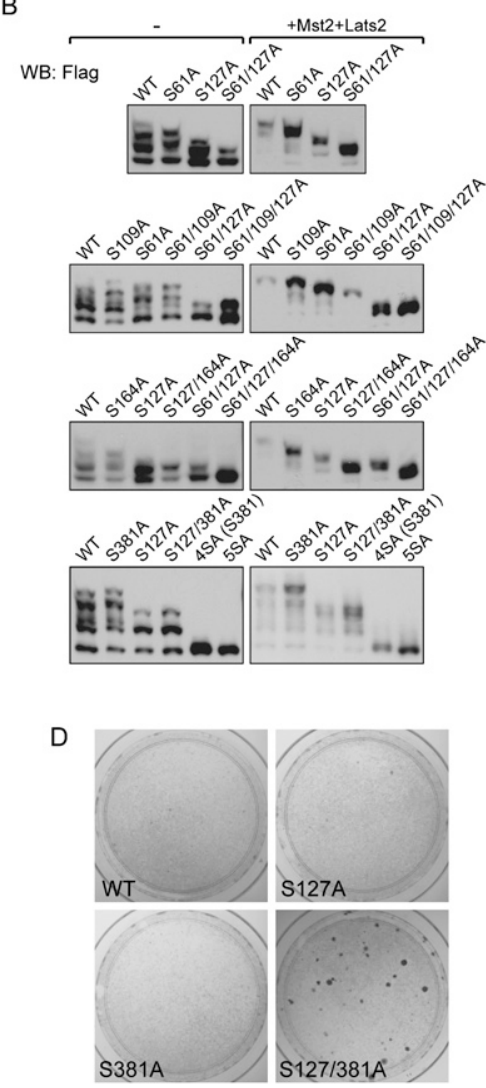

E

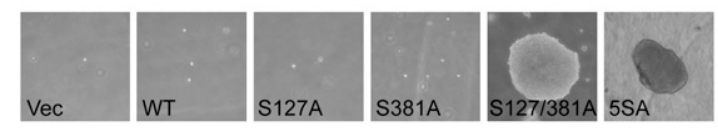

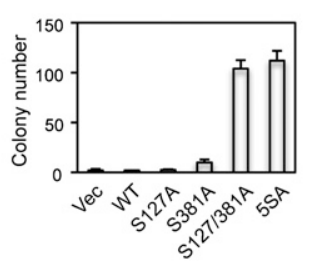

Figure 1. Phosphorylation of S127 and S381 inhibits YAP oncogenic activity. $(A)$ YAP is phosphorylated in vivo on all five Lats phosphorylation consensus sites (S61, S109, S127, S164, and S381). YAP expressed in MCF10A cells was tandem affinity-purified and analyzed by LC-MS/ MS. Identified YAP phosphopeptides were shown. Phosphorylated residues are highlighted in bold. $\left(\mathrm{M}^{\prime}\right)$ Methionine oxidation. In the phosphopeptide containing S163 and S164, the mass spectronomy data could not determine which one of them is phosphorylated. However, S164 phosphorylation was confirmed by later experiments and therefore is highlighted here. $(B)$ Mutations of S61, S109, S127, and S164 to alanine increase YAP electrophoretic mobility. YAP wild type and mutants were expressed in HEK293 cells with or without Mst and Lats cotransfection as indicated. Cell lysates were resolved on SDS-PAGE gels containing $25 \mathrm{mM}$ Phos tag-conjugated acrylamide followed by standard Western blotting with anti-Flag antibody. $(C)$ Restoration of Ser 127 or Ser 381 is sufficient to inhibit YAP-5SA transformation activity. NIH-3T3 cell colony formation assays were performed using vector control or indicated YAP constructs. Colonies were visualized with crystal violet staining 2 wk after transfection. Expression of YAP wild type or mutants $24 \mathrm{~h}$ after transfection was shown by Western blot (Supplemental Fig. S1B). (D) Mutation of both Ser 127 and Ser 381 is required to activate YAP transforming activity. Experiments were similar to those in $C$ with indicated YAP constructs. Expression of YAP wild type or mutants 24 $\mathrm{h}$ after transfection was shown by Western blot (Supplemental Fig. S1C). (E) Transformation of MCF10A cells by YAP-S127/381A and YAP-5SA. Indicated MCF10A stable cells were cultured in soft agar for $16 \mathrm{~d}$ before pictures were taken. Colonies were then visualized by crystal violet staining and colonies observable by the naked eye were counted and are shown in the right panel.

transformation, as YAP-5SA can potently transform NIH3T3 cells, while YAP-S127A is inactive (Fig. 1C,D). Therefore, at least one site among S61, S109, S164, and S381 inhibits YAP oncogenic activity. To identify this site, we restored individual serines in the YAP-5SA mutant, resulting in YAP-4SA proteins retaining a single phosphorylation site. As predicted, restoration of S127 (4SA/S127) largely inhibited colony formation (Fig. 1C). Interestingly, restoration of S381 (4SA/S381) also abolished colony formation activity, while restoration of S61 (4SA/S61), S109 (4SA/S109), and S164 (4SA/S164) did not (Fig. 1C). Therefore, restoration of either S127 or S381 is sufficient to suppress YAP oncogenic activity. As in the case of S127, mutation of S381 alone to alanine was insufficient to transform NIH-3T3 cells (Fig. 1D). Interestingly, the combination of S127 and S381 phosphorylation-deficient mutations (S127/381A) was enough to induce oncogenic transformation (Fig. 1D).
The importance of S127 and S381 in cellular transformation was also demonstrated in MCF10A cells. As shown in Figure 1E, YAP-S127/381A induced anchorageindependent growth of MCF10A cells in soft agar, as did YAP-5SA. In contrast, YAP wild type, YAP-S127A, or YAP-S381A failed to induce anchorage-independent growth. These results indicate that S127 and S381 are the two key phosphorylation sites suppressing YAP transforming activity.

\section{Ser 381 regulates YAP protein stability}

Ser 127 inhibits YAP by mediating YAP-14-3-3 binding and cytoplasmic retention upon phosphorylation (Zhao et al. 2007). However, mutation of S381 did not affect YAP interaction with 14-3-3 (Supplemental Fig. S1D). As shown in Figure 2A, YAP-S381A showed normal cytoplasmic translocation upon cotransfection with 
A

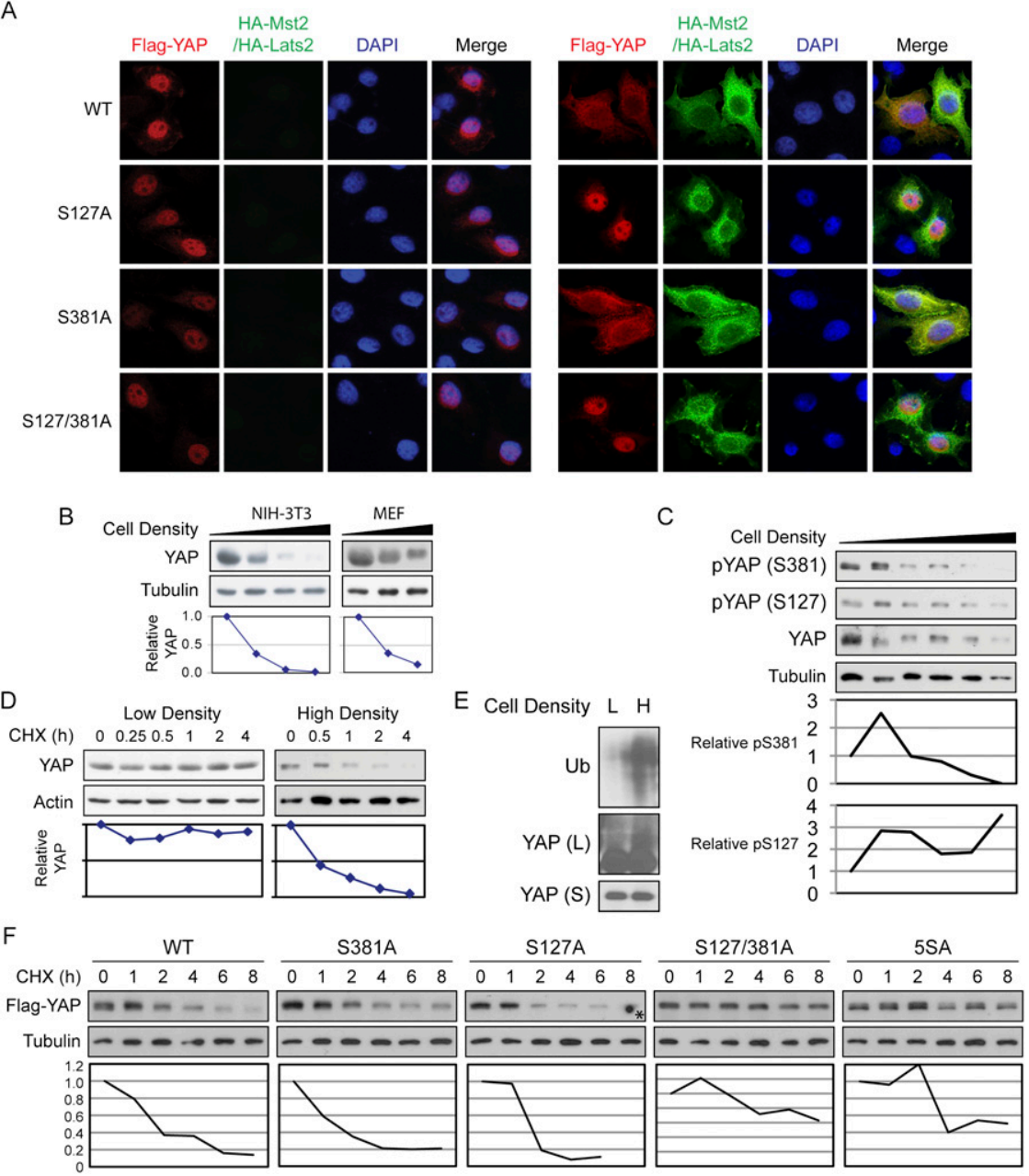

Figure 2. Mutation of Ser 381 increases YAP protein stability. (A) Mutation of S381 does not affect YAP subcellular localization. YAP wild type or mutants were expressed in HeLa cells without (left panels) or with (right panels) Mst2/ Lats2 cotransfection. YAP localization was visualized by immunofluorescence staining with antiFlag antibody (red). HA-Mst2 and HA-Lats2 were shown by anti-HA staining (green). DNA was stained with DAPI (blue). (B) YAP protein level decreases with increasing cell density. NIH-3T3 and mouse embryonic fibroblast cells were cultured at different densities from sparse to confluent. Endogenous YAP protein levels were determined by Western blot with antitubulin as a loading control. Relative YAP protein levels were quantified by the ratio between YAP and tubulin, which was arbitrarily set to 1 at the lowest cell density. $(C)$ Effect of cell density on YAP phosphorylation. NIH-3T3 cells were cultured at different densities from sparse to confluent. Endogenous YAP protein levels and YAPS381 and YAP-S127 phosphorylations were determined by Western blot with specific antibodies. Anti-tubulin was used as a loading control. Relative YAP phosphorylation levels were quantified by the ratio between pYAP and total YAP protein, which were arbitrarily set to 1 at the lowest cell density. $(D)$ YAP is unstable in high-density cell culture. NIH-3T3 cells were seeded at $1 \times 10^{6}$ per $10-\mathrm{cm}$ dish (low density) or $3 \times$ $10^{6}$ per well (high density) in six-well plates and cultured for $24 \mathrm{~h}$. Protein synthesis was blocked by treatment with $50 \mu \mathrm{g} / \mathrm{mL}$

$\mathrm{CHX}$ for the indicated time. Relative YAP protein levels were quantified by YAP/tubulin ratio, which was arbitrarily set to 1 at the 0 time point. When the same samples were resolved for a longer time, YAP showed a shift as we reported previously (Supplemental Fig. S1F) that was eliminated by $\lambda$ phosphatase treatment (Supplemental Fig. S1G). (E) YAP is ubiquitinated at high cell density. NIH-3T3 cells cultured at low or high density were treated with $25 \mu \mathrm{M} \mathrm{MG} 132$ for $5 \mathrm{~h}$ before harvest. Endogenous YAP was immunoprecipitated and Western blot was done with anti-YAP or anti-Ub antibodies. (S) Shorter exposure; (L) longer exposure. $(F)$ Mutation of S381 to alanine stabilizes YAP. NIH-3T3 cells were transfected with indicated YAP constructs. After $24 \mathrm{~h}$, cells were plated into six-well plates and cultured to confluence. Protein synthesis was blocked by treatment with $50 \mu \mathrm{g} / \mathrm{mL}$ CHX for the indicated time. Transfected YAP protein levels were shown by anti-Flag Western blot, and anti-tubulin Western blot was used as a loading control. Relative YAP protein levels were quantified by the ratio between Flag-YAP and tubulin, which was arbitrarily set to 1 at the 0 time point. The dot in the last lane of Flag-YAP-S127A Western blot, as indicated by the asterisk, was due to an artifact on the membrane, and this lane is therefore not quantified.

Mst2/Lats2. In contrast, mutation of S127 abrogated YAP cytoplasmic translocation. We also observed that, when expressed at similar levels, YAP-S381A or YAP-S127/ 381A did not exhibit significantly higher activity than wild-type or YAP-S127A protein to activate Gal4-TEAD4 in luciferase assays (Supplemental Fig. S1E). This result argues against the possibility that S381 phosphorylation directly inhibits YAP transactivation activity or TEAD binding. We set out to find out how S381 phosphorylation regulates YAP function.

We reported previously that the Hippo pathway activity increases with cell density in NIH-3T3 cell culture, which results in increased YAP phosphorylation and cytoplasmic retention (Zhao et al. 2007). We also noticed that, as cell density increases, YAP protein level declined dramatically (Fig. 2B). We ruled out the possibility that this observed decrease of YAP protein was due to interference of antibody recognition by YAP phosphorylation. In fact, the total amount of S381- or S127-phosphorylated YAP was also decreased at high density (Fig. 2C). However, when normalized to YAP protein levels, YAP S127 phosphorylation was increased, while YAP S381 phosphorylation was decreased upon cell density (Fig. 2C). Since both S127 and S381 are phosphorylated by the same kinase (Lats), a plausible explanation is that the S381phosphorylated YAP is unstable, and therefore could not 
be accumulated. The decreased YAP protein level is due, in large part, to YAP destabilization, because when protein synthesis was blocked by cycloheximide (CHX), YAP was stable at low cell density, but unstable at high cell density (Fig. 2D; Supplemental Fig. S1F). Proteasomemediated degradation of ubiquitinated protein is a common method for protein stability regulation (Hershko and Ciechanover 1998). We examined whether YAP ubiquitination is affected by cell density. As shown in Supplemental Figure S1H, YAP immunoprecipitated from proteasome inhibitor MG132-treated HA-Ub-expressing HeLa cells did exhibit incorporation of ubiquitin. Consistent with YAP destabilization at high cell density, under MG132 treatment, ubiquitinated YAP was accumulated in cells cultured at high cell density (Fig. 2E). These results indicate that activation of the Hippo pathway by cell density inhibits YAP not only by retaining YAP in cytoplasm, but also by stimulating YAP degradation.

To examine if S381 is involved in YAP degradation, we tested YAP mutant stability by CHX chase experiments. As shown in Figure 2F, similar to endogenous YAP, FlagYAP wild-type protein expressed in NIH-3T3 cells was degraded quickly at high cell density. Mutation of S127 to alanine did not stabilize YAP. Although mutation of S381 to alanine alone did not substantially stabilize YAP (Fig. 2F), when S381 was mutated in the S127A mutant background, it dramatically stabilized YAP. Similarly, the YAP-5SA mutant was also stabilized. When those YAP mutants were tested in similar experiments at low cell density, they were all stable (Supplemental Fig. S1I). These results indicate that destabilization of YAP through S381 phosphorylation is an important mecha- nism of YAP inhibition by the Hippo pathway, and this mechanism of YAP regulation is cell density-dependent.

\section{Ser 381 and the phosphodegron are required for YAP- $\beta$-TRCP interaction}

To elucidate the mechanism of S381 in the regulation of YAP stability, we carefully examined the sequence around S381 and found a DSGXS motif, similar to the canonical phosphodegron DpSGXXpS recognized by $\beta$-TRCP, an F-box-containing substrate recognition subunit in the SCF E3. Phosphorylation of the two serine residues in the phosphodegron is the key mechanism regulating $\beta$-TRCP binding and target ubiquitination (Fuchs et al. 2004). Alignment of the sequences around YAP S381 to $\beta$-catenin phosphodegron is shown in Figure $3 \mathrm{~A}$. In the case of $\beta$-catenin, S45 phosphorylation by CK1 $\alpha$ primes phosphorylation by GSK3 on T41, S37, and S33 sequentially (Liu et al. 2002; Wu and He 2006). Based on the sequence similarity between YAP and $\beta$-catenin and the function of S381 in YAP stability, we hypothesize that Lats phosphorylates S381 and primes YAP for phosphorylation by another kinase on S384 and possibly S387 in a phosphorylation relay mechanism similar to that observed in $\beta$-catenin. This sequential phosphorylation activates phosphodegron and destabilizes YAP when the Hippo pathway is activated at high cell density. It is worth noting that YAP S384 phosphorylation was also identified by mass spectrometry (Fig. 1A). Furthermore, S387 phosphorylation has also been reported by another study (Dephoure et al. 2008).

To test the above hypothesis, we first sought evidence for YAP- $\beta$-TRCP interaction. Interestingly, in our

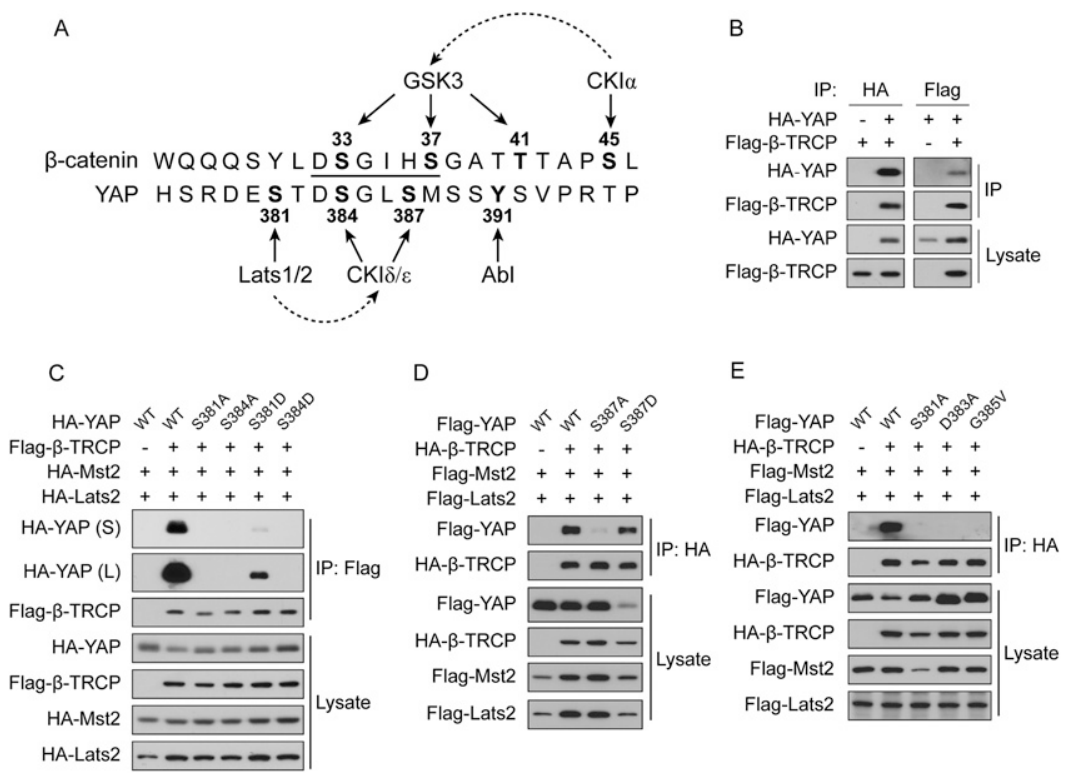

Figure 3. Ser 381 and the phosphodegron are required for YAP- $\beta$-TRCP interaction. $(A)$ Alignment of YAP sequence around S381 with the $\beta$-catenin phosphodegron sequence. The phosphodegron is underlined. Positions of phosphorylation residues are labeled. Kinases responsible (or hypothesized to be) for phosphorylation of each residue are shown. Dashed arrows indicate priming relationship. (B) YAP and $\beta$-TRCP coimmunoprecipitated with each other. Indicated plasmids were transfected into HEK293 cells. YAP or $\beta$-TRCP was immunoprecipitated with anti-HA or Flag antibody. Western blots were done to detect specific proteins as indicated on the left sides of each panel. (IP) Immunoprecipitation. (C) S381 and S384 in YAP are required for $\beta$-TRCP interaction. Indicated plasmids were transfected into HEK293 cells. $\beta$-TRCP was immunoprecipitated with anti-Flag antibody. Coimmunoprecipitated YAP was shown by anti-HA Western blot. (S) Shorter exposure; (L) longer exposure. (D) S387 is important for YAP- $\beta$-TRCP interaction. Experiments were similar to those in $C$. (E) D383 and G385 in the phosphodegron are required for YAP- $\beta$-TRCP interaction. Experiments were similar to those in $C$. 
LC-MS/MS analysis of YAP tandem affinity purification products, we did detected a $\beta$-TRCP peptide, VLEGHEELVR (peptide prophet score of 0.97), suggesting that YAP interacts with $\beta$-TRCP. This interaction was confirmed in HEK293 cells by reciprocal coimmunoprecipitation (co-IP) of HA-YAP and Flag- $\beta$-TRCP (Fig. 3B).

If S381 phosphorylation is required for YAP- $\beta$-TRCP interaction, phosphorylation-deficient mutation of this residue should abolish this interaction. As predicted, the YAP-S381A mutant lost $\beta$-TRCP binding, while the phosphomimetic mutant S381D partially retained the interaction (Fig. 3C). This result supports that YAP-S381 phosphorylation is a prerequisite for YAP- $\beta$-TRCP binding. Similarly, mutation of S384 to alanine also abolished YAP- $\beta$-TRCP interaction (Fig. $3 \mathrm{C}$ ). The function of phosphorylated S384 could not be mimicked by S384D (Fig. $3 \mathrm{C}$ ), which is not unexpected because the corresponding phosphate group in S33-phosphorylated $\beta$-catenin directly forms hydrogen bonds with $\beta$-TRCP /G Wu et al. 2003 ), and therefore an acidic residue at this position may not substitute for the function of a phosphate group.

A classical phosphodegron has three or more residues between the two phosphorylated serines (Fuchs et al. 2004). In the case of YAP, there are only two residues between S384 and S387 (Fig. 3A). To test whether S387 phosphorylation is also important for YAP- $\beta$-TRCP binding, we examined co-IP of S387A and S387D mutants with $\beta$-TRCP. Mutation of S387 to alanine significantly reduced YAP- $\beta$-TRCP interaction (Fig. 3D), indicating the importance of this residue. Moreover, the S387D mutant largely retained the ability of $\beta$-TRCP binding (Fig. 3D), suggesting that S387 phosphorylation contributes to $\beta$-TRCP interaction. In the case of $\beta$-catenin, mutation of the conserved D and G residues in the phosphodegron found in cancer patients attenuates $\beta$-TRCP interaction and ubiquitination (Provost et al. 2005). The equivalent YAP mutants, D383A and G385V, also lost YAP- $\beta$-TRCP interaction (Fig. 3E). The above results strongly suggest that YAP contains a phosphodegron. The interaction between YAP and $\beta$-TRCP depends on phosphorylation of the serine residues 381, 384, and 387, and also the intact DSG motif in the phosphodegron.

\section{The Hippo pathway stimulates YAP-S381/384 phosphorylation and $\beta$-TRCP interaction}

To study the regulation of YAP-S381 and YAP-S384 phosphorylation and the relationship with YAP- $\beta$-TRCP interaction, we generated a phosphorylation-specific antibody against pYAP (S381/384). As shown in Figure $4 \mathrm{~A}$, this antibody has only minimal recognition of phosphorylation-deficient YAP-S381A or YAP-S384A mutants compared with YAP wild type. Furthermore, $\lambda$ protein phosphatase treatment abolished recognition of wild-type YAP by this antibody, thus confirming its specificity (Fig. 4A). Using this antibody, we showed that coexpression of Mst2 and Lats2 enhanced phosphorylation on these residues, while coexpression of Mst2 and Lats2 dominantnegative mutants inhibited YAP phosphorylation (Fig. 4B). Regulation of $S 381 / 384$ phosphorylation by the
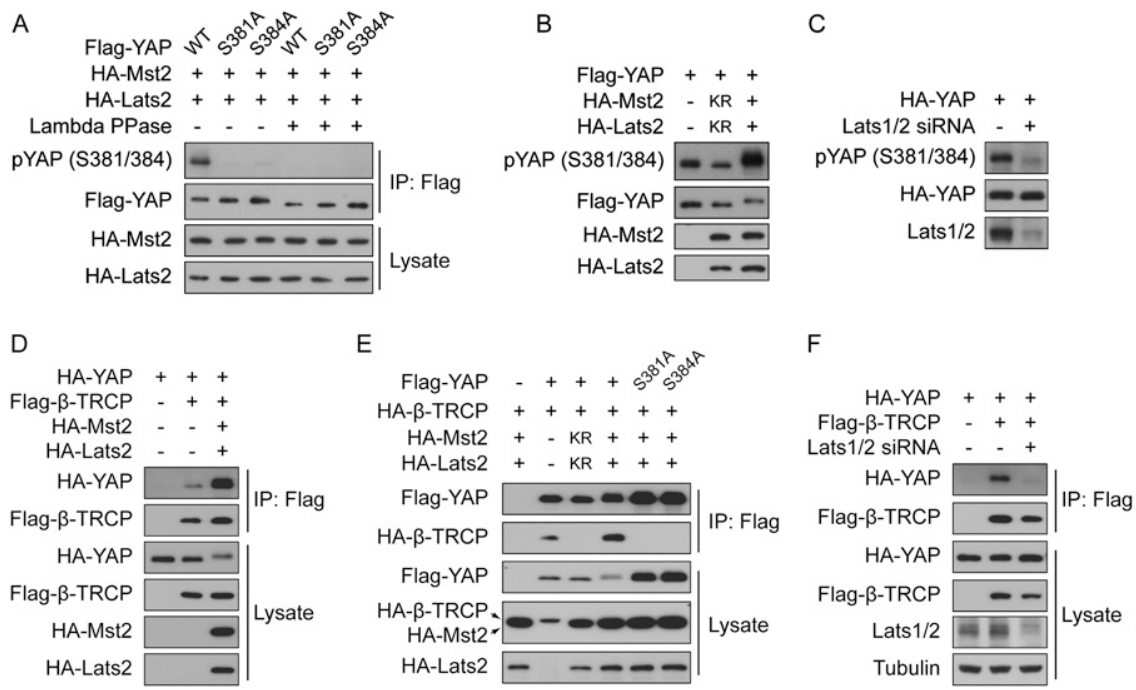

Figure 4. The Hippo pathway induces YAP S381 phosphorylation and YAP$\beta$-TRCP interaction. (A) pYAP (S381/384) antibody preferentially recognizes S381/ 384-phosphorylated YAP. YAP wild type or mutants were coexpressed with Mst2 and Lats2 in HEK293 cells. Flag-YAP was then immunoprecipitated and one set of the immunoprecipitates was treated with $\lambda$ protein phosphatase as indicated. The immunoprecipitated YAP phosphorylation and protein levels were then detected by anti-pYAP (S381/384) antibody and antiFlag antibody, respectively. (B) Mst2 and Lats2 induce YAP-S381/384 phosphorylation. Indicated plasmids were cotransfected into HEK293 cells. (KR)Kinase-inactive mutants. YAP phosphorylation was shown by a pYAP (S381/384)-specific antibody. (C) Lats knockdown significantly attenuates YAP-S381/384 phosphorylation. HA-YAP was expressed in HEK293 cells. Lats1 and Lats2 knockdown was achieved by two rounds of siRNA transfection with 24-h intervals. YAP phosphorylation was shown by a pYAP (S381/384)-specific antibody. Anti-HA Western blot indicates YAP protein level. Lats knockdown efficiency was shown by an antibody against both Lats1 and Lats2. $(D)$ Mst2 and Lats2 stimulate YAP- $\beta$-TRCP interaction. Indicated plasmids were transfected into HEK293 cells. $\beta$-TRCP was immunoprecipitated with anti-Flag antibody, and coimmunoprecipitated YAP was shown by anti-HA Western blot. (E) Dominant-negative Mst2 and Lats2 inhibit YAP- $\beta$-TRCP interaction. Indicated plasmids were transfected into HEK293 cells. (KR) Kinase-inactive mutants. YAP was immunoprecipitated with anti-Flag antibody, and coimmunoprecipitated $\beta$-TRCP was shown by anti-HA Western blot. $(F)$ Lats knockdown inhibits YAP- $\beta$-TRCP interaction. Indicated plasmids were cotransfected with or without Lats1/2 siRNA. Lats1/2 siRNA transfection was repeated once after $24 \mathrm{~h}$. $\beta$-TRCP was immunoprecipitated with anti-Flag antibody, and coimmunoprecipitated YAP was shown by anti-HA Western blot. Lats knockdown efficiency was shown by an antibody against both Lats1 and Lats2. 
Hippo pathway was further confirmed by a significant decrease of YAP-S381/384 phosphorylation upon Lats $1 / 2$ knockdown (Fig. 4C).

Consistent with the up-regulation of S381/384 phosphorylation, Mst2 and Lats 2 cotransfection also dramatically enhanced YAP- $\beta$-TRCP interaction, as shown by co-IP experiments (Fig. 4D). The effect of Mst2 and Lats2 was also seen by a reciprocal co-IP (Fig. 4E). Moreover, expression of dominant-negative Mst2 and Lats2 inhibited YAP- $\beta$-TRCP interaction (Fig. 4E). Finally, knockdown of Lats $1 / 2$ also strongly inhibited YAP- $\beta$-TRCP binding (Fig. 4F), while this inhibition was rescued by expressing RNAi-resistant mouse Lats2 wild-type protein, but not the kinase-inactive form (Supplemental Fig. S2), supporting a role of endogenous Lats in regulating YAP interaction with $\beta$-TRCP. The above results confirm our hypothesis that the Hippo pathway promotes S381/ 384 phosphorylation, and therefore YAP- $\beta$-TRCP interaction. S381 in the Lats phosphorylation consensus is directly phosphorylated by Lats, while S384 is likely to be phosphorylated by another kinase.

S381 phosphorylation by Lats primes YAP

for a sequential phosphorylation by $C K 1 \delta / \varepsilon$

in the phosphodegron and subsequent $\beta$-TRCP binding

According to our hypothesis, the kinase responsible for S384 phosphorylation may require priming phosphoryla- tion on -3 position serine (S381), and the same kinase may be responsible for S387 phosphorylation. Interestingly, the CK1 family kinases are known to have a canonical consensus of pS/T-X ${ }_{1-2}-\mathrm{S} / \mathrm{T}$ (Knippschild et al. 2005). We tested whether CK1 is involved in S384 phosphorylation by using a general CK1 inhibitor (D4476) and a CK1 $\delta / \varepsilon$-specific inhibitor (IC261). Both inhibitors strongly decreased YAP interaction with $\beta$-TRCP (Supplemental Fig. S3A), and, as shown in Figure 5A, IC261 blocked YAP phosphorylation as detected by the pYAP (S381/384)-specific antibody, supporting a role of CK1 $\delta / \varepsilon$ in S384 phosphorylation. This was further confirmed by coexpression of CK1 $1 \varepsilon$ with YAP. Wild-type but not the kinase-inactive CK1 $\varepsilon$ increased recognition by anti-pYAP (S381/384) antibody by $40 \%$ (Fig. 5B).

To confirm the relationship between Lats phosphorylation on S381 and the subsequent CK1 $/ \delta / \varepsilon$ phosphorylation on serine residues 384 and 387, we performed a twostep phosphorylation reaction in vitro. GST-YAP substrates were first subjected to Lats2 kinase assay reaction with cold ATP followed by removal of Lats2. The Lats2pretreated GST-YAP was subsequently phosphorylated by CK1 1 kinase in the presence of ${ }^{32} \mathrm{P}$-ATP. As expected, without $\mathrm{CK} 1 \varepsilon$ treatment, no ${ }^{32} \mathrm{P}$ incorporation was detected in GST-YAP wild type (Fig. 5C). After the two-step kinase reaction, GST-YAP was efficiently phosphorylated by CK1 1 (as indicated by the incorporation of ${ }^{32} \mathrm{P}$ ). The
A

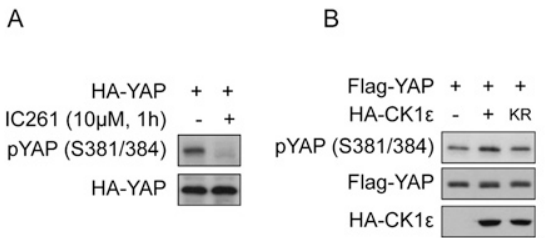

D

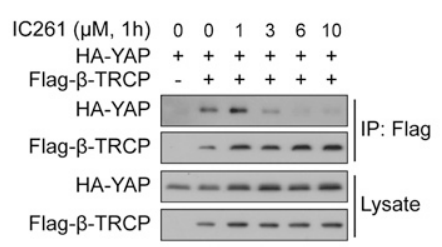

C

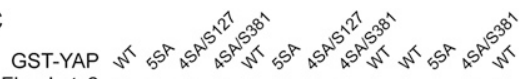

Flag-Lats2 + + + + + + + - - +

HA-CK1ع - - - + + + + + + KR
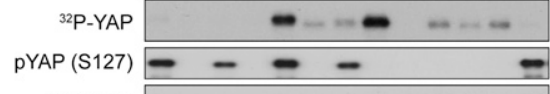

GSTYYAP

Flag-Lats2 $--\infty-\infty-\infty$

${ }^{32} \mathrm{P}-\mathrm{CK} 1 \varepsilon$

HA-CK1ع

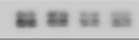

$-\cdots-\cdots$

E

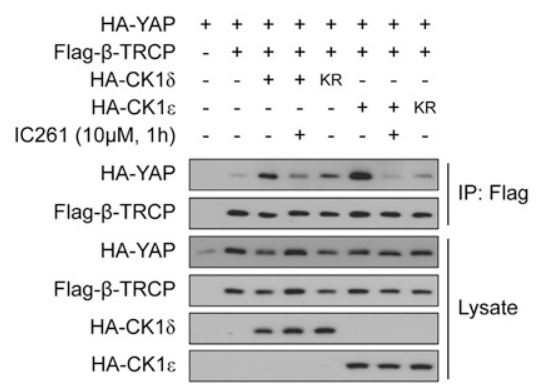

Figure 5. YAP-S381 phosphorylation primes it for S384 phosphorylation by CK1 $\delta / \varepsilon$ and stimulates $\beta$-TRCP binding. (A) YAP phosphorylation is inhibited by CK $1 \delta / \varepsilon$ inhibitor. HA-YAP was transfected into HEK293 cells. Cells were treated with CK $1 \delta / \varepsilon$ inhibitor IC261, and phosphorylation of S381 and S384 was detected in Western blot by a phosphospecific antibody. (B) CK1s increases YAP-S384 phosphorylation in a kinase activity-dependent manner. HEK293 cells were transfected and Western blot was done as indicated. (KR) Kinase-inactive form of CK1 1 . Quantification of the ratio between pYAP (S381/384) and Flag-YAP showed a $40 \%$ increase of the phosphorylation by coexpression of CK1ع. (C) In vitro phosphorylation of YAP by CK1 1 requires S381 priming phosphorylation by Lats. Flag-Lats 2 and HA-CK1\& were expressed in HEK293 cells separately and were immunoprecipitated with resin conjugated with anti-Flag or antiHA antibody. GST-YAP substrates were expressed and purified from Escherichia coli and were first subjected to Lats kinase assay with cold ATP as indicated. Aliquots of the

reaction mixture supernatant (without the resin-bound Lats kinase) were taken to the second-step kinase assay with immunoprecipitated HA-CK $1 \varepsilon$ and ${ }^{32}$ P-ATP. Resin after the first-step reaction was prepared for Western blot for Lats protein level. All autoradiography and other Western blots were from samples after the second-step reaction. $(D)$ CK1 $\delta / \varepsilon$ inhibitor dose-dependently inhibited the interaction between YAP and $\beta$-TRCP. HA-YAP and Flag- $\beta$-TRCP were expressed in HEK293 cells as indicated. Cells were treated with the indicated concentration of IC261 for $1 \mathrm{~h}$ before harvest. Flag- $\beta$-TRCP was immunoprecipitated, and coimmunoprecipitated YAP was shown by antiHA Western blot. $(E)$ CK1 $\delta / \varepsilon$ increases YAP- $\beta$-TRCP interaction in a kinase activity-dependent manner. HEK293 cells were transfected with indicated plasmids and were treated with IC261 as indicated. Flag- $\beta$-TRCP was immunoprecipitated, and coimmunoprecipitated YAP was shown by anti-HA Western blot. 
kinase-inactive CK1 $1 \varepsilon-\mathrm{KR}$ mutant failed to phosphorylate GST-YAP, supporting a direct role of CK1 1 in GST-YAP phosphorylation. Furthermore, without the pretreatment with Lats2, GST-YAP was minimally phosphorylated by CK1 1 . Together, these data demonstrate the importance of Lats priming phosphorylation for CK1 $1 \varepsilon$ to phosphorylate YAP.

To further test the requirement of S381 for YAP phosphorylation by $\mathrm{CK} 1 \varepsilon$, various YAP mutants were tested. As expected, phosphorylation of GST-YAP by CK1 $1 \varepsilon$ was largely diminished if the YAP-5SA mutant was used as a substrate (Fig. 5C), and was not rescued by restoration of the S127 site (4SA/S127) (Fig. 5C; Supplemental Fig. S3B). In contrast, restoration of S381 in the 5SA mutant (4SA/ S381) completely rescued YAP phosphorylation by CK1 $\varepsilon$. The above results further support our model that phosphorylation of S381 by Lats is a prerequisite for YAP-S384 and possibly YAP-S387 phosphorylation by CK1 $1 \varepsilon$.

We then examined the function of CK $1 \delta / \varepsilon$ on YAP$\beta$-TRCP interaction. Consistent with the inhibition of S384 phosphorylation (Fig. 5A), IC261 dose-dependently inhibited the interaction between YAP and $\beta$-TRCP (Fig. $5 \mathrm{D})$. To rule out the possibility that IC261 inhibits YAP$\beta$-TRCP interaction by nonspecifically inhibiting the Hippo pathway, we examined the effect of IC261 on the interaction of $\beta$-TRCP with YAP mutant 4SA/S381 or S381D. When four out of the five Lats phosphorylation sites, except S381, were mutated in YAP, its interaction with $\beta$-TRCP was similarly inhibited by IC261 (Supplemental Fig. S3C), indicating the effect of IC261 is not mediated by the other four HXRXXS sites. When S381 was mutated to a phosphomimetic aspartic residue (S381D), IC261 still inhibited the interaction between YAP (S381D) and $\beta$-TRCP (Supplemental Fig. S3C). Moreover, the inhibition of YAP- $\beta$-TRCP interaction by IC261 was also observed when the Hippo pathway was activated by coexpression of Mst2 and Lats2 (Supplemental Fig. S3D). The above results ruled out the possibility that the inhibitory effect of IC261 on the interaction between YAP and $\beta$-TRCP is due to inhibition of the Hippo pathway, thus supporting our model that $\mathrm{CK} 1 \delta / \varepsilon$ phosphorylation of $\mathrm{S} 384$ is required for YAP- $\beta$-TRCP binding. Consistently, increase of YAP- $\beta$-TRCP interaction was observed by coexpression of CK1 $1 \delta$ or CK $1 \varepsilon$ (Supplemental Fig. S3E). This stimulation of YAP- $\beta$-TRCP interaction depends on CK $1 \delta / \varepsilon$ kinase activity, as the effect of CK $1 \delta / \varepsilon$ was blocked by IC261 treatment and the kinase-inactive CK1 $1 / \varepsilon$ had little effect (Fig. 5E). The above results support our model that, following S381 phosphorylation by Lats,

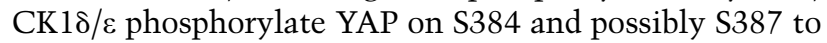
activate the phosphodegron and permit $\beta$-TRCP binding.

\section{YAP ubiquitination depends on phosphorylation of S381 and phosphodegron}

YAP ubiquitination was elevated when cells were cultured at high density (Fig. 2E), a condition under which the Hippo pathway is activated. We tested if YAP ubiquitination is stimulated by the Hippo pathway kinases and $\mathrm{SCF}^{\beta-\mathrm{TRCP}}$. Indeed, expression of Mst2 and Lats2 induced
Myc-Ub incorporation and a ladder pattern of YAP protein when proteasome function was inhibited by MG132 (Fig. 6A). Coexpression of the SCF ${ }^{\beta-T R C P}$ complex components ( $\beta$-TRCP, SKP1, and Cul1) further increased YAP ubiquitination in a manner dependent on Mst/Lats coexpression. These results support the functional importance and collaboration of Mst/Lats and $\mathrm{SCF}^{\beta-T R C P}$ in stimulating YAP ubiquitination.

We next examined the function of S381 and phosphodegron in YAP ubiquitination. As shown in Figure 6B, the phosphorylation-deficient S381A mutant largely lost its ubiquitination. However, when the same residue was mutated to a phosphomimetic S381D, which partially restored $\beta$-TRCP binding (Fig. 3C), YAP ubiquitination was also partially rescued (Fig. 6B). The above results confirm the importance of S381 phosphorylation in YAP ubiquitination. When D383 and S384, two key residues in the phosphodegron, were mutated to alanine, YAP ubiquitination was abolished, highlighting the essential role of the phosphodegron in YAP ubiquitination. Mutation of S387 to alanine (S387A) showed a lower degree in reduction of YAP ubiquitination, while S387D mutation rescued YAP ubiquitination (Fig. 6B). The behavior of these two mutants is completely consistent with their effect on YAP- $\beta$-TRCP binding (Fig. 3D), further supporting that S387 is important but not essential for YAP ubiquitination. We also tested the function of CK1 $\delta / \varepsilon$ on YAP ubiquitination by using the inhibitor IC261. As shown in Figure 6C, IC261 inhibited Myc-Ub incorporation into YAP, supporting an important function of endogenous CK1 $\delta / \varepsilon$ in YAP ubiquitination.

$S C F^{\beta-T R C P}$-mediated YAP degradation is stimulated

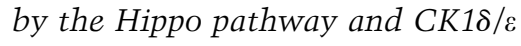

To confirm that YAP stability is regulated by the $S_{C F}{ }^{\beta-T R C P}$, we examined the effect of blocking $S^{3} F^{\beta-T R C P}$ function by a dominant-negative Cull. As shown by CHX chase, YAP is unstable at high cell density. However, YAP was stabilized when dominant-negative Cull was coexpressed (Fig. 7A). This supports that the $\mathrm{SCF}^{\beta-T R C P}$ promotes YAP degradation. At low cell density, the Hippo pathway is inactive and YAP is stable (no significant degradation under CHX chase up to $6 \mathrm{~h}$ ) (Fig. 7B). However, if the Hippo pathway was activated by expressing Mst2 and Lats2 in low-density cells, YAP was destabilized (Fig. 7B). Similarly, expression of CK1 $\varepsilon$ also destabilized YAP. However, the S127/381A mutant was not significantly destabilized by Mst2/Lats2 or CK1ع (Fig. 7B). These observations indicate a role of Mst/Lats and $\mathrm{CK} 1 \delta / \varepsilon$ in promoting YAP degradation.

We observed that mutation of the Lats phosphorylation site S381 stabilized YAP, especially in the S127A background (Fig. 2F), and the mutant was resistant to Mst2-, Lats2-, and CK1 $\varepsilon$-induced degradation (Fig. 7B). We also examined the CK $1 \delta / \varepsilon$ phosphorylation site S384 and found that S384A mutation in the S127A background dramatically stabilized YAP (Fig. 7C). Similar observation was made with another key phosphodegron residue mutant, D383A (Fig. 7C). If a protein is actively degraded by 
Zhao et al.

A

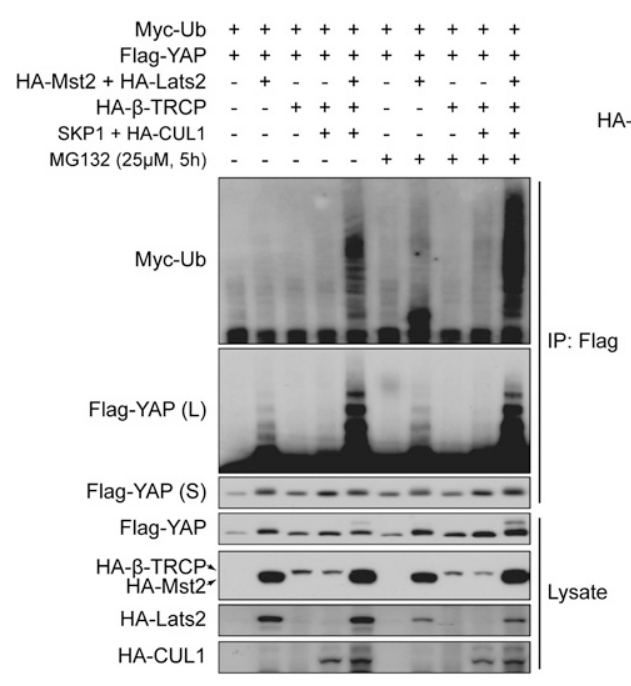

C

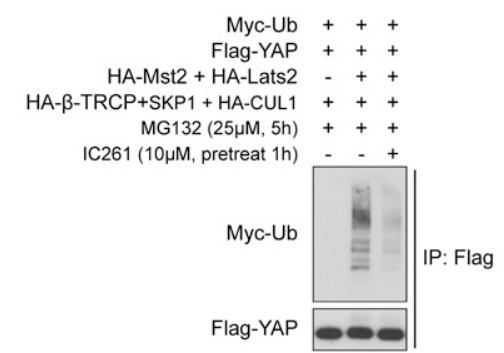

B

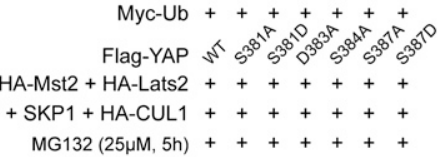

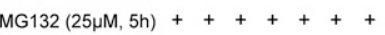

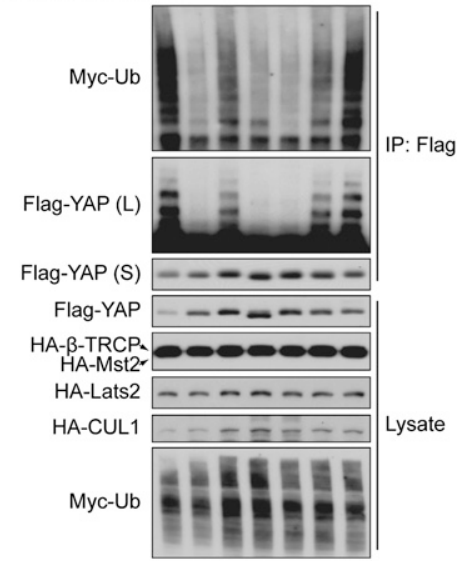

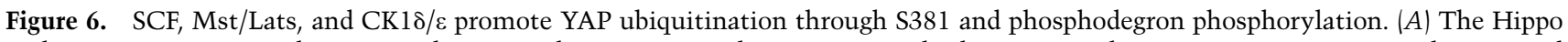
pathway components and SCF stimulate YAP ubiquitination. Flag-YAP, Myc-Ub, the Hippo pathway components Mst2 and Lats2, and the SCF complex components $\beta$-TRCP, SKP1, and CUL1 were cotransfected into HEK293 cells as indicated. Cells were treated with MG132 as indicated before harvest. Flag-YAP was immunoprecipitated, and Western blots were done with specific antibodies. (L) longer exposure for Flag-YAP; (S) shorter exposure for Flag-YAP. (B) YAP ubiquitination is dependent on S381, 384, and 387 phosphorylation and on intact phosphodegron. YAP wild type and mutants were cotransfected with other plasmids into HEK293 cells as indicated. Cells were treated with $25 \mu \mathrm{M}$ MG132 for $5 \mathrm{~h}$ before harvest. Flag-YAP was immunoprecipitated, and Western blots were done with indicated antibodies. $(C)$ YAP ubiquitination is inhibited by the CK1 $\delta / \varepsilon$ inhibitor IC261. HEK293 cells were transfected as indicated. Cells were pretreated with $10 \mu \mathrm{M}$ IC261 for $1 \mathrm{~h}$ and then treated with MG132 as indicated before harvest. Flag-YAP was immunoprecipitated, and Western blots were done with indicated antibodies.

proteasome, blocking proteasome function should result in accumulation of this protein. Indeed, MG132 treatment induced a steady accumulation of YAP protein when cells were cultured at high cell density (Fig. 7D). However, when the Hippo pathway activity was inhibited by dominantnegative Mst2 and Lats2, the MG132-induced accumulation of YAP was not observed, indicating that the Hippo pathway is required for YAP degradation. Similarly, muta-

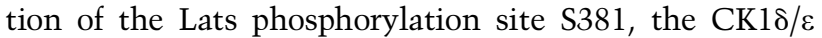
phosphorylation site S384, or a key phosphodegron residue D383 on the S127A background diminished MG132induced YAP accumulation (Fig. 7D). Together, the above results strongly support the regulation of YAP stability by the Hippo pathway, CK1 $1 \delta / \varepsilon$, and the phosphodegron.

\section{The phosphodegron suppresses YAP oncogenic} transformation activity

Finally, we investigated the function of the CK $1 \delta / \varepsilon$ phosphorylation site S384 and the phosphodegron in regulating
YAP oncogenic activity. As shown in Figure 7E, YAP with a single-site mutation of S127, S381, S384, S387, or D383 was unable to transform NIH-3T3 cells. However, similar to the S127/381A double mutant, the S127/384A and S127/D383A double mutants could transform NIH-3T3 cells as potently as the 5SA protein (Fig. 7E). The S127/ 387A double mutant could also transform NIH3T3 cells, though less actively, consistent with the less stringent requirement of S387 for $\beta$-TRCP binding. Collectively, our data support the importance of S381 priming phosphorylation by Lats, the subsequent S384 and S387 phosphorylation by $\mathrm{CK} 1 \delta / \varepsilon$, and the phosphodegron recognition by $\mathrm{SCF}^{\beta-T R C P}$ in repression of YAP oncogenic activity.

\section{Discussion}

Spatial and temporal regulation of YAP

Accumulating evidence supports the role of YAP as a key controller of organ size and as a human oncogene. 
A

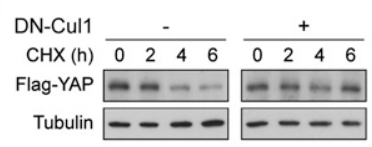

B

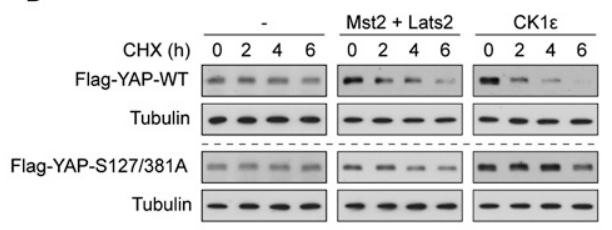

C

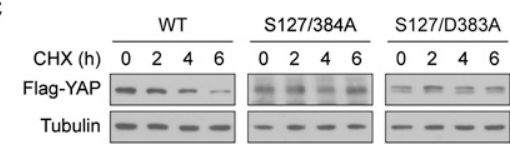

D

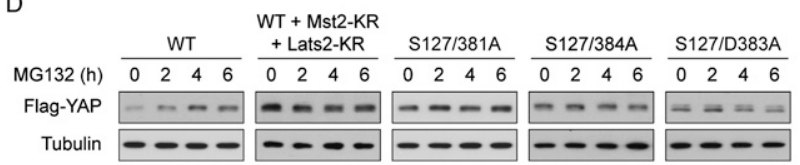

E
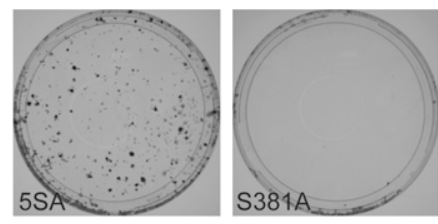

si27a

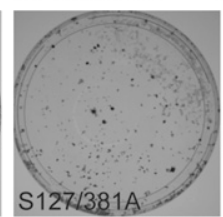

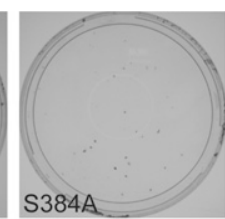
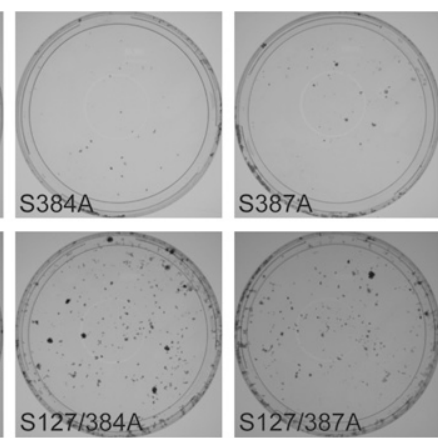

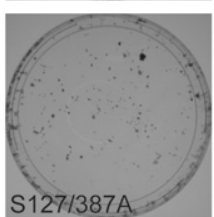

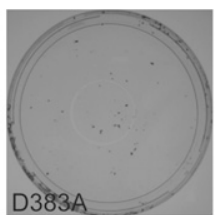

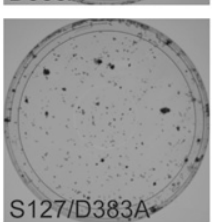

Figure 7. Inhibition of YAP oncogenic transformation ability through $\mathrm{SCF}^{\beta-T R C P}$-mediated YAP degradation depends on the Hippo pathway, CK $1 \delta / \varepsilon$, and the phosphodegron. (A) YAP degradation is inhibited by dominant-negative Cul1. Flag-YAP was transfected into NIH-3T3 cells at low expression level alone lleft panels) or together with dominant-negative Cull (right panels), and cells were cultured to confluence. Cells were treated with $50 \mu \mathrm{g} / \mathrm{mL}$ $\mathrm{CHX}$ for the indicated time. Anti-Flag Western blot was used to show YAP protein levels and anti-tubulin Western blot was used as a loading control. $(B)$ The Hippo pathway and CK1 1 destabilize YAP in a S381-dependent manner. Flag-YAP wild type or S127/381A mutant were transfected alone or together with the Hippo pathway components Mst2/ Lats2, or CK1 $\varepsilon$ into NIH-3T3 cells. Cells were cultured at low density so that most cells do not have contact with each other. CHX chase and Western blots were done as in A. (C) Mutation of S384 or D383 in the phosphodegron stabilizes YAP. YAP wild type or mutants were transfected into NIH-3T3 cells. Cells were cultured to confluence, and CHX chase and Western blots were done as in $A$. (D). Regulation of YAP stability by proteasome is dependent on functional Hippo pathway, as well as S381 and the phosphodegron of YAP. Flag-YAP wild type or mutants were transfected alone or together with kinaseinactive Mst2-KR and Lats2-KR as indicated. Cells were cultured to confluence and were treated with $25 \mu \mathrm{M}$ MG132 for the indicated time before harvest. Western blots were done as in $A$. $(E)$ Mutation of the phosphodegron together with S127 is sufficient for YAP to transform NIH-3T3 cells. NIH-3T3 cell colony formation assays were performed using indicated YAP constructs. Colonies were visualized with crystal violet staining.

Elucidating the mechanisms regulating YAP activity will have implications in the normal physiology of organ size regulation and pathogenesis of human cancer. The Hippo pathway is the only inhibitor of YAP known to date. It has been shown to play a key role in limiting organ size in Drosophila (Pan 2007), and deregulation of several components of this pathway, such as NF2 mutation (McClatchey and Giovannini 2005), has been implicated in human cancer. Previously, we showed that the Hippo pathway inhibits YAP by S127 phosphorylation-mediated 14-3-3 binding and cytoplasmic retention (Zhao et al. 2007), therefore providing a mechanism of spatial separation of YAP from its nuclear target transcription factors, such as TEAD (Fig. 8).

YAP has been shown recently to be ubiquitinated, although the mechanism was unknown (Lapi et al. 2008). The data we presented in this study elucidated another layer of YAP regulation. By phosphorylation on S381, the Hippo pathway primes YAP for phosphorylation by CK $1 \delta / \varepsilon$, and subsequent ubiquitination and degradation (Fig. 8). This provides a mechanism of temporal regulation of YAP protein levels upon activation of the Hippo pathway. Under physiological conditions like high cell density, the S381 phosphorylation-mediated degradation might be the major cause for YAP degradation. As shown in Figure 2C, relative S381 phosphorylation dropped dramatically when cell density increased, although relative S127 phosphorylation of YAP was increased, indicating that the S381phosphorylated YAP could not be accumulated, possibly due to degradation. However, it is possible that there exists a S127 phosphorylation-dependent fail-safe mechanism for YAP destabilization when S381-mediated degradation is not working properly. Such a mechanism may explain why both S127 and S381 mutations are required for YAP stabilization. Our study reveals that inhibition of YAP by the Hippo pathway is more complex than expected, with both spatial and temporal mechanisms. We speculate that the spatial regulation could provide a reversible short-term inhibition of YAP, while the temporal regulation through YAP degradation may provide an irreversible long-term inhibition. Dysregulation of both mechanisms could lead to oncogenic transformation.

It is worth noting that the S381-initiated degradation of YAP is not conserved in Drosophila Yki, because this phosphorylation site and the phosphodegron are not present in Yki, although they are conserved through vertebrates. However, this does not exclude the possibility that Yki protein stability is controlled by the Hippo 


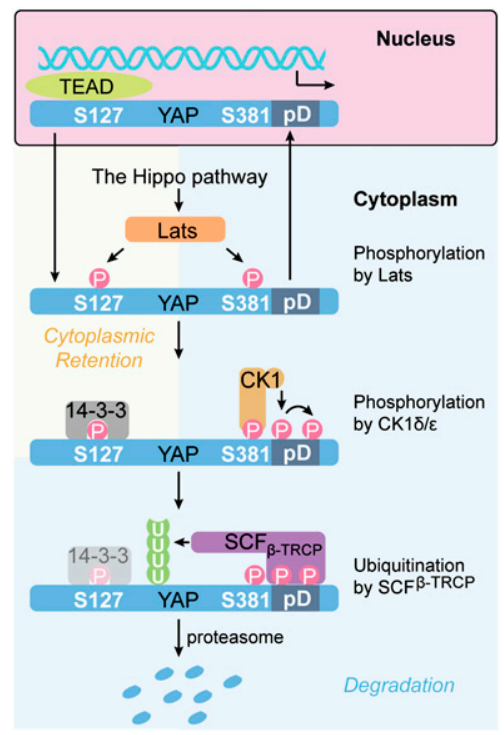

Figure 8. A model of YAP inhibition by the Hippo pathway and CK1. When the Hippo pathway is activated, YAP is phosphorylated on both S127 and S381 in the HXRXXS motifs. Phosphorylation of S127 results in 14-3-3 binding and cytoplasmic retention of YAP. Therefore, YAP can be inhibited by spatial separation from its nuclear target transcription factors, such as TEAD. This mechanism of regulation is reversible. Phosphorylation of S381 primes YAP phosphorylation by CK $1 \delta / \varepsilon$, resulting in activation of a phosphodegron, which then recruits the $\mathrm{SCF}^{\beta-\mathrm{TRCP}} \mathrm{E} 3$ ubiquitin ligase, leading to YAP degradation. This mechanism of regulation is irreversible. Through these two mechanisms, YAP activity is under both spatial and temporal control, which coordinately inhibit YAP oncogenic activity.

pathway through other mechanisms. The phosphodegron is conserved in TAZ, a YAP paralog, and also modulates TAZ stability in a similar manner (QYL and KL Guan, unpubl.).

Are there additional mechanisms of YAP regulation by the Hippo pathway? The possibility exists. Our studies confirmed three other Lats phosphorylation sites in YAP, but their functions are unknown. Although these sites do not seem to play an obvious role in controlling the oncogenic activity of YAP, as indicated by NIH-3T3 cell transformation assay, they may function in other contexts.

\section{Lessons from $\beta$-catenin for the regulation of YAP}

The similarity between YAP and $\beta$-catenin is quite interesting. $\beta$-catenin is also a transcription coactivator implicated in malignant transformation. Without Wnt signaling, $\beta$-catenin is constantly degraded through $\mathrm{SCF}^{\beta-\mathrm{TRCP}}$. mediated ubiquitination (Clevers 2006). Similar to YAP, $\beta$-catenin binding with SCF $^{\beta-T R C P}$ depends strictly on multistep phosphorylation of the phosphodegron involving CK1 $\alpha$ and GSK-3 (Liu et al. 2002). Perturbation of this process leads to $\beta$-catenin accumulation in colorectal cancer, HCCs, and malignant melanomas (Frescas and Pagano 2008). There are similarities between YAP and $\beta$-catenin in many aspects, including their function as transcription coactivators with growth-promoting activity and as latent oncogenes. They are both subject to multistep phosphorylation and phosphodegron-dependent ubiquitination by $\mathrm{SCF}^{\beta-\mathrm{TRCP}}$, and deregulation of the degradation leads to oncogenic transformation.

Extensive studies have been done to analyze mutations leading to $\beta$-catenin stabilization, which should shed light on future studies of YAP. In the case of $\beta$-catenin, its stabilization in cancer is frequently due to failure to recruit GSK3 as a result of inactivating mutations of adenomatous polyposis coli (APC) or axin (Rubinfeld et al. 1996; Liu et al. 2000). In some cases, stabilization of $\beta$-catenin also results from mutation in the phosphodegron and its priming phosphorylation sites (Morin et al. 1997). Interestingly, elevated YAP protein levels have been observed in some cancers (Zender et al. 2006; Dong et al. 2007; Zhao et al. 2007; Steinhardt et al. 2008). It will be interesting to survey possible YAP mutations in cancer samples and identify proteins regulating YAP phosphodegron phosphorylation. It will also be important to examine deregulation of YAP protein levels as a result of Hippo pathway component mutations in cancer.

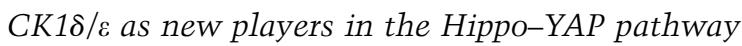

CK1 is a family of multifunctional kinases with unique substrate specificity as $\mathrm{pS} / \mathrm{T}-\mathrm{X}_{1-2}-\mathrm{S} / \mathrm{T}$ (Knippschild et al. 2005). Phosphorylation by CK1 requires preceding phosphorylation of residue at the -2 or -3 position of the target residue. This requirement of a priming phosphorylation by another kinase provides a possible mechanism of signal integration in complex biological processes. For example, in the case of YAP destabilization, the requirement of CK $1 \delta / \varepsilon$ phosphorylation following Lats phosphorylation may integrate other signals besides the Hippo pathway to regulate YAP. CK1 is often referred to as constitutively active kinase. However, it has also been reported that CK1 is regulated by subcellular localization and inhibitory autophosphorylation by stimuli such as $\gamma$ irradiation and Wnt signaling (Knippschild et al. 2005). At high cell density, we observed a clear drop of relative YAP-S381 phosphorylation and an increase of relative YAP-S127 phosphorylation. The fact that both sites are phosphorylated by Lats kinase suggests that phosphorylation of S384 might induce YAP degradation. It will be interesting to investigate if cell density increases CK1 activity.

In Drosophila, the CK $1 \delta / \varepsilon$ homolog discs overgrown $(d c o)$ has been positioned in the Hippo pathway upstream of dachs by its regulation of the Hippo pathway downstream target genes and by genetic epistasis experiments (Cho et al. 2006). Recently, dco has further been shown to phosphorylate Fat (Sopko et al. 2009), although it has not been determined if this phosphorylation directly affects Fat function and the Hippo pathway activity. However, the function of CK1 $1 \delta / \varepsilon$ in regulating YAP- $\beta$-TRCP interaction is not due to inhibition of the Hippo pathway, as both YAP-4SA/S381 and YAP-S381D mutants are still inhibited by IC261. Conversely, the mechanism of CK1 $1 / \varepsilon$ in regulating YAP stability is unlikely to be conserved in 
$d c o$, as the phosphodegron is not conserved in Yki. Nevertheless, the function of $d c o / C K 1 \delta / \varepsilon$ in inhibiting Yki/YAP is conserved between Drosophila and mammals, although different mechanisms may be employed.

\section{The phosphodegron of YAP}

YAP contains a phosphodegron, DSGXS, that is highly similar to but does not exactly match the canonical DSGXXS phosphodegron. However, the requirement of the second serine residue for $\beta$-TRCP binding is less stringent compared with the first one. In the reported phosphodegron variants, some of them require the second serine to be further away from the DSG (Frescas and Pagano 2008), and, in certain cases like CDC25A, the second $\mathrm{S}$ is not even required (Jin et al. 2003). In the case of YAP, the second serine (S387) is not absolutely required, but contributes to YAP interaction with $\beta$-TRCP and YAP ubiquitination. This was shown by the residual binding between $\beta$-TRCP and the phosphorylationdeficient S387A, and the largely normal binding between $\beta$-TRCP and the phosphomimetic S387D (Fig. 3D).

The exact YAP sequence $S_{(-3)} \operatorname{TDS}_{(0)} G$, where $S_{(-3)}$ (S381) serves as a priming phosphorylation site for $\mathrm{S}_{(0)}$ (S384), is conserved in some other $\beta$-TRCP substrates like CDC25A, which contains $\mathrm{S}_{(-6)} \mathrm{XXS}_{(-3)} \mathrm{TDS}_{(0)} \mathrm{G}$ (Supplemental Fig. S4). In this case, the -6 position serine phosphorylation by Chk 1 is shown to be required for CDC25A binding with $\beta$-TRCP and subsequent degradation in vivo (Jin et al. 2003). However, in an in vitro binding assay, a peptide with phosphorylation on the $S_{(0)}$ but not $S_{(-3)}$ showed a strong binding to $\beta$-TRCP, which was not further enhanced by phosphorylation on $S_{(-3)}$ (Jin et al. 2003). This in vitro binding assay using peptides sharing similar phosphodegron structure with YAP helps us to exclude the function of YAP-S381 as an integral part of the phosphodegron directly involved in $\beta$-TRCP binding, but rather supports $S 381$ as a priming phosphorylation site for S384 phosphorylation by CK1 $1 \delta / \varepsilon$. Compared with YAP, we speculate that the main function of the $\mathrm{S}_{(-3)}$ in the CDC25A phosphodegron might be a phosphorylation-relaying residue passing the signal from the -6 position to the 0 position instead of being directly involved in $\beta$-TRCP binding. Phosphodegron with a phosphorylated -3 position serine also exists in other known SCF $^{\beta-T R C P}$ substrates, such as RE-1 silencing transcription factor (REST) (Supplemental Fig. S4; Westbrook et al. 2008). Together with YAP, they may represent a class of SCF $^{\beta-T R C P}$ substrates containing a SXDSG phosphodegron, in which the first serine serves as a priming phosphorylation site. In the case of CDC25A and REST, the kinase responsible for phosphorylating the second serine residue is unknown. The CK1 family kinases are attractive candidates for this function because of their $\mathrm{pS} /$ $\mathrm{T}-\mathrm{X}_{1-2}-\mathrm{S} / \mathrm{T}$ target consensus. We speculate that there may be a broader role for the CK1 family in $\mathrm{SCF}^{\beta-\mathrm{TRCP}}$. mediated protein ubiquitination and degradation.

In close proximity with the YAP phosphodegron, there is a tyrosine residue (Y391) reported to be phosphorylated by c-Abl in response to DNA damage, which results in
YAP stabilization (Levy et al. 2008). Future studies are needed to test if the Y391 phosphorylation modulates $S_{C F}{ }^{\beta-T R C P}$-mediated YAP ubiquitination and degradation.

\section{Materials and methods}

\section{Immunofluorescence staining}

Cells were cultured on coverslips to the appropriate density. Cells were fixed with $4 \%$ paraformaldehyde for $15 \mathrm{~min}$ and then permeabilized with $0.1 \%$ Triton X-100. After blocking in 3\% bovine serum albumin (BSA) for $30 \mathrm{~min}$, slides were incubated with first antibody diluted in $1 \%$ BSA for $1.5 \mathrm{~h}$. After washing with PBS, slides were incubated with Alexa Fluor 488- or 594conjugated secondary antibodies (1:1000 dilution) for $1.5 \mathrm{~h}$. The slides were then washed and mounted.

\section{Immunoprecipitation and kinase assay}

For the Lats2 and CK1 1 two-step kinase assays, HEK293 cells were transfected with Flag-Lats2 or HA-CK1ع. Forty-eight hours post-transfection, cells were lysed with lysis buffer (50 mM HEPES at $\mathrm{pH} 7.5,150 \mathrm{mM} \mathrm{NaCl}, 1 \mathrm{mM}$ EDTA, $1 \%$ NP-40, $10 \mathrm{mM}$ pyrophosphate, $10 \mathrm{mM}$ glycerophosphate, $50 \mathrm{mM}$ $\mathrm{NaF}, 1.5 \mathrm{mM} \mathrm{Na}_{3} \mathrm{VO}_{4}$, protease inhibitor cocktail [Roche], $1 \mathrm{mM}$ DTT, $1 \mathrm{mM}$ PMSF), and immunoprecipitated with anti-Flag or anti-HA antibody-conjugated resin. The immunoprecipitates were washed three times with lysis buffer, and then once with wash buffer (40 mM HEPES, $200 \mathrm{mM} \mathrm{NaCl}$ ) and once with kinase assay buffer (30 mM HEPES, $50 \mathrm{mM}$ potassium acetate, $5 \mathrm{mM} \mathrm{MgCl}_{2}$ ). In each reaction, $1 \mu \mathrm{g}$ of GST-YAP2, which was expressed and purified from Escherichia coli, was first subjected to Lats2 kinase assay in the presence of the immunoprecipitated Lats 2 and $500 \mu \mathrm{M}$ cold ATP. The reaction mixtures were incubated for $30 \mathrm{~min}$ at $30^{\circ} \mathrm{C}$, and then briefly incubated on ice followed by centrifugation and removal of an aliquot of the supernatant to the next-step reaction. The leftover with the resin was supplemented with SDS loading buffer and cooked to examine the Lats2 protein level. Supernatants from the first step were then supplemented with immunoprecipitated HA-CK $1 \varepsilon$ and 10 $\mu \mathrm{Ci}\left(\gamma^{32} \mathrm{P}\right)$ ATP. The mixtures were incubated for $30 \mathrm{~min}$ at $30^{\circ} \mathrm{C}$, and the reactions were terminated with SDS sample buffer and subjected to SDS-PAGE and autoradiography.

\section{RNAi}

Smart pool siRNA oligonucleotides toward human Lats1 or Lats2 and control siRNA toward firefly luciferase were purchased from Dharmacon. siRNAs were transfected into HEK293 cells twice with a $24-\mathrm{h}$ interval. Cell lysate was made $48 \mathrm{~h}$ posttransfection.

\section{Colony formation assay}

Colony formation assay was performed as described briefly below. NIH-3T3 fibroblasts were seeded on six-well plates at a density of $10^{5}$ cells per well and then transfected with YAP wild type or mutants using FuGene6 (Roche) according to the manufacturer's instructions. After $2 \mathrm{~d}$, cells were trypsinized and an aliquot of cells were lysed for YAP expression; other cells were replated onto a $10-\mathrm{cm}$ dish and were maintained in DMEM supplemented with $5 \%$ fetal bovine serum for $2-3$ wk until foci were evident. Cells were fixed with $10 \%$ acetic acid and $10 \%$ methanol, and then colonies were stained with $1 \%$ crystal violet and counted. 


\section{Acknowledgments}

We thank Drs. Philip Gafken at the Fred Hutchinson Cancer Center for assistance in LC-MS/MS analyses; Joungmok Kim for preparation of GST-YAP proteins; Yue Xiong for HA-Ub-stableexpressing HeLa cells and pCMV-Flag- $\beta$-TRCP, pcDNA3-HACul1, pcDNA3-SKP1, and PCW7-Myc-His-Ub plasmids; Stephen J. Elledge for DN-Cul1 plasmid; and Xi He for CK1 $\delta$ and CK1 $1 \varepsilon$ plasmids. This work was supported by grants from the NIH (to K.L.G.).

\section{References}

Camargo FD, Gokhale S, Johnnidis JB, Fu D, Bell GW, Jaenisch $\mathrm{R}$, Brummelkamp TR. 2007. YAP1 increases organ size and expands undifferentiated progenitor cells. Curr Biol 17: 2054-2060.

Cho E, Feng Y, Rauskolb C, Maitra S, Fehon R, Irvine KD. 2006. Delineation of a Fat tumor suppressor pathway. Nat Genet 38: 1142-1150.

Clevers H. 2006. Wnt/ $\beta$-catenin signaling in development and disease. Cell 127: 469-480.

Dephoure N, Zhou C, Villen J, Beausoleil SA, Bakalarski CE, Elledge SJ, Gygi SP. 2008. A quantitative atlas of mitotic phosphorylation. Proc Natl Acad Sci 105: 10762-10767.

Deshaies RJ. 1999. SCF and Cullin/Ring H2-based ubiquitin ligases. Annu Rev Cell Dev Biol 15: 435-467.

Dong J, Feldmann G, Huang J, Wu S, Zhang N, Comerford SA, Gayyed MF, Anders RA, Maitra A, Pan D. 2007. Elucidation of a universal size-control mechanism in Drosophila and mammals. Cell 130: 1120-1133.

Frescas D, Pagano M. 2008. Deregulated proteolysis by the F-box proteins SKP2 and $\beta$-TrCP: Tipping the scales of cancer. Nat Rev Cancer 8: 438-449.

Fuchs SY, Spiegelman VS, Kumar KG. 2004. The many faces of $\beta$-TrCP E3 ubiquitin ligases: Reflections in the magic mirror of cancer. Oncogene 23: 2028-2036.

Hao Y, Chun A, Cheung K, Rashidi B, Yang X. 2008. Tumor suppressor LATS1 is a negative regulator of oncogene YAP. I Biol Chem 283: 5496-5509.

Hart M, Concordet JP, Lassot I, Albert I, del los Santos R, Durand H, Perret C, Rubinfeld B, Margottin F, Benarous R, et al. 1999. The F-box protein $\beta$-TrCP associates with phosphorylated $\beta$-catenin and regulates its activity in the cell. Curr Biol 9: 207-210.

Harvey KF, Pfleger CM, Hariharan IK. 2003. The Drosophila Mst ortholog, hippo, restricts growth and cell proliferation and promotes apoptosis. Cell 114: 457-467.

Hershko A, Ciechanover A. 1998. The ubiquitin system. Annu Rev Biochem 67: 425-479.

Huang J, Wu S, Barrera J, Matthews K, Pan D. 2005. The Hippo signaling pathway coordinately regulates cell proliferation and apoptosis by inactivating Yorkie, the Drosophila homolog of YAP. Cell 122: 421-434.

Jia J, Zhang W, Wang B, Trinko R, Jiang J. 2003. The Drosophila Ste20 family kinase dMST functions as a tumor suppressor by restricting cell proliferation and promoting apoptosis. Genes \& Dev 17: 2514-2519.

Jin J, Shirogane T, Xu L, Nalepa G, Qin J, Elledge SJ, Harper JW. 2003. SCF $\beta$-TRCP links Chk1 signaling to degradation of the Cdc25A protein phosphatase. Genes \& Dev 17: 30623074.

Kango-Singh M, Nolo R, Tao C, Verstreken P, Hiesinger PR, Bellen HJ, Halder G. 2002. Shar-pei mediates cell proliferation arrest during imaginal disc growth in Drosophila. Development 129: 5719-5730.
Knippschild U, Gocht A, Wolff S, Huber N, Lohler J, Stoter M. 2005. The casein kinase 1 family: Participation in multiple cellular processes in eukaryotes. Cell Signal 17: 675-689.

Lai ZC, Wei X, Shimizu T, Ramos E, Rohrbaugh M, Nikolaidis N, Ho LL, Li Y. 2005. Control of cell proliferation and apoptosis by mob as tumor suppressor, mats. Cell 120: 675-685.

Lapi E, Di Agostino S, Donzelli S, Gal H, Domany E, Rechavi G, Pandolfi PP, Givol D, Strano S, Lu X, et al. 2008. PML, YAP, and $\mathrm{p} 73$ are components of a proapoptotic autoregulatory feedback loop. Mol Cell 32: 803-814.

Latres E, Chiaur DS, Pagano M. 1999. The human F box protein $\beta$-Trcp associates with the Cul1/Skp1 complex and regulates the stability of $\beta$-catenin. Oncogene 18: 849-854.

Levy D, Adamovich Y, Reuven N, Shaul Y. 2008. Yap1 phosphorylation by $\mathrm{c}-\mathrm{Abl}$ is a critical step in selective activation of proapoptotic genes in response to DNA damage. Mol Cell 29: 350-361.

Li Q, Verma IM. 2002. NF-кB regulation in the immune system. Nat Rev Immunol 2: 725-734.

Liu W, Dong X, Mai M, Seelan RS, Taniguchi K, Krishnadath KK, Halling KC, Cunningham JM, Boardman LA, Qian C, et al. 2000. Mutations in AXIN2 cause colorectal cancer with defective mismatch repair by activating $\beta$-catenin/TCF signalling. Nat Genet 26: 146-147.

Liu C, Li Y, Semenov M, Han C, Baeg GH, Tan Y, Zhang Z, Lin $X, H e X$. 2002. Control of $\beta$-catenin phosphorylation/degradation by a dual-kinase mechanism. Cell 108: 837-847.

McClatchey AI, Giovannini M. 2005. Membrane organization and tumorigenesis-the NF2 tumor suppressor, Merlin. Genes \& Dev 19: 2265-2277.

Morin PJ, Sparks AB, Korinek V, Barker N, Clevers H, Vogelstein B, Kinzler KW. 1997. Activation of $\beta$-catenin-Tcf signaling in colon cancer by mutations in $\beta$-catenin or APC. Science 275: 1787-1790.

Oh H, Irvine KD. 2009. In vivo analysis of Yorkie phosphorylation sites. Oncogene 28: 1916-1927.

Oka T, Mazack V, Sudol M. 2008. Mst2 and Lats kinases regulate apoptotic function of Yes kinase-associated protein (YAP). J Biol Chem 283: 27534-27546.

Overholtzer M, Zhang J, Smolen GA, Muir B, Li W, Sgroi DC, Deng CX, Brugge JS, Haber DA. 2006. Transforming properties of YAP, a candidate oncogene on the chromosome $11 \mathrm{q} 22$ amplicon. Proc Natl Acad Sci 103: 12405-12410.

Pan D. 2007. Hippo signaling in organ size control. Genes \& Dev 21: 886-897.

Pantalacci S, Tapon N, Leopold P. 2003. The Salvador partner Hippo promotes apoptosis and cell-cycle exit in Drosophila. Nat Cell Biol 5: 921-927.

Pickart CM. 2001. Mechanisms underlying ubiquitination. Annu Rev Biochem 70: 503-533.

Provost E, McCabe A, Stern J, Lizardi I, D'Aquila TG, Rimm DL. 2005. Functional correlates of mutation of the Asp32 and Gly34 residues of $\beta$-catenin. Oncogene 24: 2667-2676.

Rubinfeld B, Albert I, Porfiri E, Fiol C, Munemitsu S, Polakis P. 1996. Binding of GSK3 $\beta$ to the APC- $\beta$-catenin complex and regulation of complex assembly. Science 272: 10231026.

Sopko R, Silva E, Clayton L, Gardano L, Barrios-Rodiles M, Wrana J, Varelas X, Arbouzova NI, Shaw S, Saburi S, et al. 2009. Phosphorylation of the tumor suppressor fat is regulated by its ligand Dachsous and the kinase discs overgrown. Curr Biol 19: 1112-1117.

Steinhardt AA, Gayyed MF, Klein AP, Dong J, Maitra A, Pan D, Montgomery EA, Anders RA. 2008. Expression of Yesassociated protein in common solid tumors. Hum Pathol 39: $1582-1589$. 
Tapon N, Harvey KF, Bell DW, Wahrer DC, Schiripo TA, Haber DA, Hariharan IK. 2002. salvador Promotes both cell cycle exit and apoptosis in Drosophila and is mutated in human cancer cell lines. Cell 110: 467-478.

Udan RS, Kango-Singh M, Nolo R, Tao C, Halder G. 2003. Hippo promotes proliferation arrest and apoptosis in the Salvador/ Warts pathway. Nat Cell Biol 5: 914-920.

Westbrook TF, Hu G, Ang XL, Mulligan P, Pavlova NN, Liang A, Leng Y, Maehr R, Shi Y, Harper JW, et al. 2008. SCF $\beta$-TRCP controls oncogenic transformation and neural differentiation through REST degradation. Nature 452: 370-374.

Wu G, He X. 2006. Threonine 41 in $\beta$-catenin serves as a key phosphorylation relay residue in $\beta$-catenin degradation. Biochemistry 45: 5319-5323.

Wu G, Xu G, Schulman BA, Jeffrey PD, Harper JW, Pavletich NP. 2003. Structure of a $\beta$-TrCP1-Skp1- $\beta$-catenin complex: Destruction motif binding and lysine specificity of the SCF $(\beta-\operatorname{TrCP} 1)$ ubiquitin ligase. Mol Cell 11: 1445-1456.

Wu S, Huang J, Dong J, Pan D. 2003. hippo encodes a Ste-20 family protein kinase that restricts cell proliferation and promotes apoptosis in conjunction with salvador and warts. Cell 114: 445-456.

Zender L, Spector MS, Xue W, Flemming P, Cordon-Cardo C, Silke J, Fan ST, Luk JM, Wigler M, Hannon GJ, et al. 2006. Identification and validation of oncogenes in liver cancer using an integrative oncogenomic approach. Cell 125: 12531267.

Zeng Q, Hong W. 2008. The emerging role of the hippo pathway in cell contact inhibition, organ size control, and cancer development in mammals. Cancer Cell 13: 188-192.

Zhang I, Smolen GA, Haber DA. 2008. Negative regulation of YAP by LATS1 underscores evolutionary conservation of the Drosophila Hippo pathway. Cancer Res 68: 2789-2794.

Zhao B, Wei X, Li W, Udan RS, Yang Q, Kim J, Xie J, Ikenoue T, $\mathrm{Yu}$ J, Li L, et al. 2007. Inactivation of YAP oncoprotein by the Hippo pathway is involved in cell contact inhibition and tissue growth control. Genes \& Dev 21: 2747-2761.

Zhao B, Ye X, Yu J, Li L, Li W, Li S, Lin JD, Wang CY, Chinnaiyan AM, Lai ZC, et al. 2008. TEAD mediates YAPdependent gene induction and growth control. Genes \& DeV 22: 1962-1971.

Zhao B, Kim J, Ye X, Lai ZC, Guan KL. 2009. Both TEADbinding and WW domains are required for the growth stimulation and oncogenic transformation activity of yesassociated protein. Cancer Res 69: 1089-1098. 


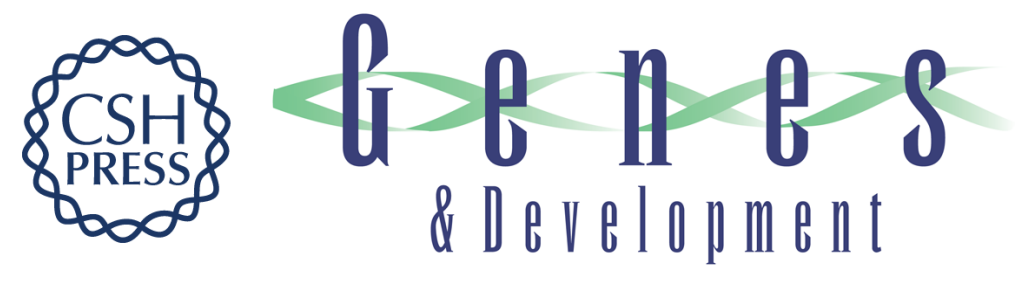

\section{A coordinated phosphorylatipn by Lats and CK1 regulates YAP stability through SCF $\beta$-TRCP}

Bin Zhao, Li Li, Karen Tumaneng, et al.

Genes Dev. 2010, 24:

Access the most recent version at doi:10.1101/gad.1843810

Supplemental
Material http://genesdev.cshlp.org/content/suppl/2009/12/14/24.1.72.DC1

References This article cites 48 articles, 14 of which can be accessed free at: http://genesdev.cshlp.org/content/24/1/72.full.html\#ref-list-1

License

Email Alerting

Receive free email alerts when new articles cite this article - sign up in the box at the top Service right corner of the article or click here.

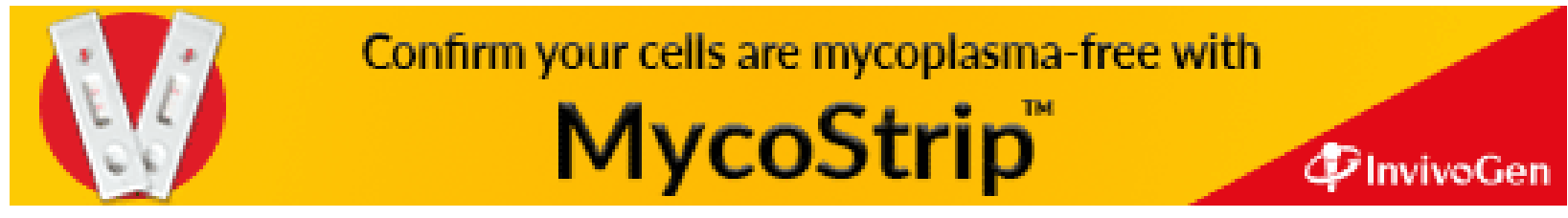

